\title{
Modified UWB Spatio-Temporal Channel Simulation Including Pulse Distortion and Frequency Dependence
}

\author{
Khajitpan Makaratat, Tim W. C. Brown, Member, IEEE, and Stavros Stavrou, Member, IEEE
}

\begin{abstract}
A modified simulation of ultra-wideband (UWB) multipath channels, combined with cluster classification and physicsbased pulse distortion mechanisms, is proposed in this letter. Spatiotemporal characteristics of multipath clusters are specifically generated based on $3 \times 3$ planar array systems with regard to scenario types and are simulated over ten frequency subbands (2-11 GHz). Thus, frequency-dependent characteristics of the propagation channels are also investigated and compared between each scenario. Finally, the probability of the bit-error rate is determined to quantify distortion effects on UWB multipath channels for all frequency subbands.
\end{abstract}

Index Terms-Distortion quantification, pulse distortion, spatio-temporal channel simulation, ultra-wideband (UWB) frequency dependence.

\section{INTRODUCTION}

$\mathbf{T}$ HERE are extensive works in channel investigations that discuss ultra-wideband (UWB) multipath clusters and their dependency on measurement bandwidth and the considered environment. In general, the UWB multipath cluster model is described by the classical Saleh-Valenzuela (SV) model [1] and by some modifications as proposed by Chong et al. [2] and Spencer et al. [3] where, in the latter, the combined spatio-temporal statistical model for indoor multipath propagation was presented herein. In order to investigate UWB channel modeling, its propagation paths are characterized as the stationary condition where the channel characteristics remain constant within finite space, time, and frequency intervals. However, most of the current available UWB channel model simulations imply only channel impulse responses (CIRs) without any details of obstruction clusters and distortion effects to support realistic UWB propagation channels. Cheng and Dubey proposed a generic statistical channel model for a wideband environment [4]. This work mentioned the cluster classification and frequency subbands in the simulation process. The departure and arrival multipath rays were grouped into a cluster in which a set of angles of arrival (AOAs), angles of departure (AODs), and times of arrival (TOAs) were generated for each frequency interval. This can gain UWB multipath characteristics for each considered frequency subband.

Manuscript received May 25, 2008; May 30, 2008. First published June 10, 2008; current version published December 30, 2008.

The authors are with the Centre for Communication Systems Research, University of Surrey, Surrey GU2 7XH, U.K. (e-mail: K.Makaratat@ surrey.ac.uk).

Digital Object Identifier 10.1109/LAWP.2008.2001174
Essentially, in order to investigate the realistic UWB spatio-temporal channel statistics and the geometrically based pulse distortion model, the proposed simulation is a combination of simulating characteristics of UWB multipath clusters and physics-based pulse distortions captured by planar arrays. In addition to time domain characteristics, this simulation is extended to include the frequency-dependent electromagnetic properties of obstruction materials. The capability of generating accurate site-specific UWB CIRs and their realistic pulse waveforms, which contain distortion, can be provided. Alternatively, it also gains a viable and affordable way to investigate the UWB CIRs with the advantage of short simulation time due to generating only specific dominant multipath signals. This is less time consuming compared with the ray-tracing technique in which all propagation paths are computed. Finally, UWB receiving system performance is also presented to quantify distortion effects. All cases of multipath channels are taken into account over all frequency subbands.

\section{UWB Multipath Clusters AND Distortion CHANNEL IMPULSE RESPONSE}

\section{A. Classification of UWB Multipath Clusters}

According to multipath cluster investigations derived from many indoor UWB measurements [1]-[3], [5], [6], each multipath cluster could be identified by a group of multipath components (MPCs). The received MPCs from dominant propagation paths from a transmitter to a receiver are expected to come from three types of radio propagation paths. The first group corresponds to scattering near the transmitter site, Class-I clusters. Similarly, Class-II clusters can be observed at the receiver site due to the scattering objects in the neighboring area of the receiver. Finally, line-of-sight (LOS) components between the transmitter and the receiver are considered as Class-III clusters [4]. In addition to CIRs to be employed, distortion characteristics are also considered for constructing the realistic UWB channel model. According to UWB channel properties for multipath environments, more than one multipath ray can be observed within a short time bin [1], [7]. These overlapped ray arrivals can inevitably cause pulse-shape distortions. As a result, the simulation technique presented in this letter also includes a UWB time-domain distortion model based on a geometric-optic (GO) technique to describe direct and reflected rays, and based on the geometric/uniform theory of diffraction (GTD/UTD) technique to describe the diffracted rays, including the perfectly conducting half-plane diffraction 


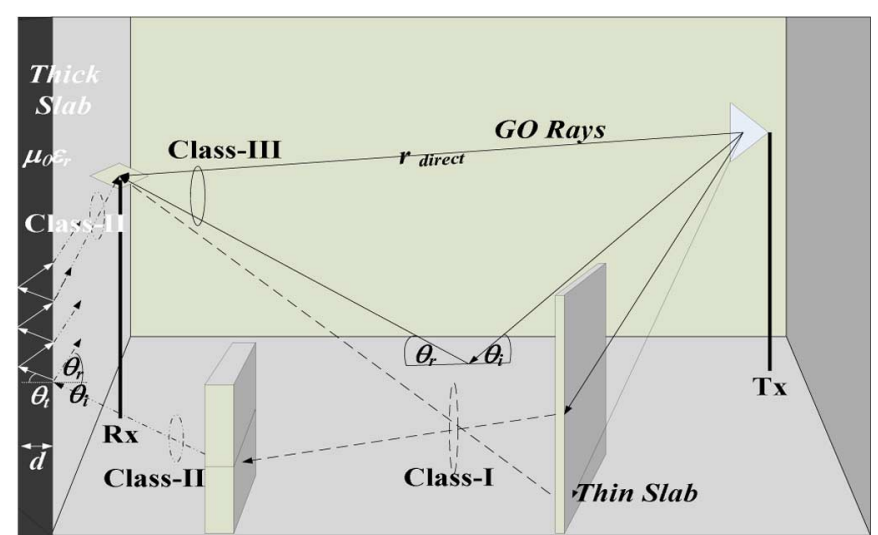

Fig. 1. Illustration of UWB propagation clusters and distortion mechanisms.

and propagation through d-thickness-slab obstructions [7], [8]. The illustration of classified UWB clusters and pulse distortion mechanisms is presented in Fig. 1.

\section{B. Physics-Based-Distorted CIR Modeling}

Consequently, in order to construct realistic UWB propagation channels, classified MPC clusters and pulse distortion effects are simulated together. Typically, the total response $h$ $(\tau)$ from a complex multipath channel can be modeled by the summation of all impulse responses of local scattering with the closed-form expression of specific geometric configurations. Unlike typical UWB channel models [9], this simulation relies on combining pulse distortion characteristics into the UWB CIR model. In addition, this modified simulation also includes frequency-dependent effects as each CIR model is considered at each frequency subband $s$. For instance, in the LOS scenario of Case-A describing a small furnished office room, the channel would be dominated by Class-III clusters and distorted by multiple reflections and half-plane diffraction as defined by

$$
\begin{aligned}
& h_{\text {case- } A}(s, \tau, \theta, \phi)=h_{\mathrm{LOS}}^{\mathrm{III}}(s, \tau, \theta, \phi)+h_{\mathrm{GO}}^{\mathrm{III}}(s, \tau, \theta, \phi) \\
& \quad+h_{\text {half-plane }}^{\text {III }}(s, \tau, \theta, \phi) .
\end{aligned}
$$

Next, the NLOS scenario Case-B is considered where a transmitter and a receiver are in the same office room with a-light-wall or a-cloth-partition separation between both ends; the channel would be still dominated by Class-III clusters and distorted by the similar effects as Case-A. However, thin slab diffraction included as an addition into the distortion model $h_{\text {thin-slab }}^{\mathrm{III}}(s, \tau, \theta, \phi)$ to construct $h_{\text {case- } B}$. When propagation paths in a larger furnished office room NLOS Case-C are considered, all three classes of clusters are presented. All distortion effects might possibly appear in Class-III similar to LOS-A, but distortions due to thin-slab diffraction and thick-slab reflection are modeled for Class-II clusters. The CIRs of Case-C can be described by (2) where $g^{I}\left(s, \theta_{T x}, \sigma_{T x}\right)$ is the single-directional CIR of Class-I clusters at the transmitter site. $\theta_{T x}$ and $\phi_{T x}$ are the elevation and azimuth AOD of the MPCs

$$
\begin{aligned}
& h_{\text {case- } C}(s, \tau, \theta, \phi)=h_{\mathrm{GO}}^{\mathrm{II}}(s, \tau, \theta, \phi)+h_{\text {half-plane }}^{\mathrm{III}}(s, \tau, \theta, \phi) \\
& \quad+h_{\text {thin-slab }}^{\mathrm{II}}(s, \tau, \theta, \phi) \\
& \quad+h_{\text {thick-slab }}^{\mathrm{II}}(s, \tau, \theta, \phi)+g^{I}\left(s, \theta_{T x}, \phi_{T x}\right) .
\end{aligned}
$$

Finally, if a transmitter and a receiver are located in a different furnished room or separated by a thick wall, the channel would be dominated by Class-I and Class-II clusters corresponding to the extreme NLOS condition (Case-D). Only thick-slab reflection is considered. Thus, only the last two terms in (2) are taken into account for this $h_{\text {case- } D}$. Since this paper concentrates on novel frequency-dependent results obtained from the proposed simulation, all technical terms of $h_{\mathrm{LOS}}, h_{\mathrm{GO}}, h_{\text {half-plane, }}, h_{\text {thin-slab }}$, and $h_{\text {thick-slab }}$ are not illustrated in this letter, but their formulas are written in detail as defined in [4], [7], and [8].

\section{Modified Spatiotemporal SimUlation AND FREQUENCY-DEPENDENT RESULTS}

\section{A. Modified Spatiotemporal Simulation}

This section proposes a UWB pulse simulation method based on planar array systems, a different approach than the one proposed in [4]. Moreover, this modified simulation also incorporates the physics-based pulse distortion model by using priorimodeled parameters [1]-[3], [5], [6] as presented in Table I with a uniform distribution, $\mathrm{U}[:,:]$, denoted in some parameters. The entire frequency $(2-11 \mathrm{GHz})$ is divided into ten subbands with each subband occupying 1-GHz bandwidth. CIRs and distorted pulses are generated to represent the arrival rays regarding UWB characteristics. The UWB transmitted pulse consists of the second derivative of a Gaussian function with 1-ns pulse width. Types and numbers of dominated clusters corresponding to each specific scenario are also described in Table I. Parameter sets of $\beta_{\phi}, \gamma_{\phi}$, and $\beta_{\theta}, \gamma_{\theta}$ are used to calculate angle spread (AS) to determine the AOAs and AODs in azimuth and elevation angles. $\beta_{\tau}$ and $\gamma_{\tau}$ are used to compute delay spread (DS) in order to form TOAs. The number of clusters and MPCs arriving within each cluster correspond to cluster and ray decay factors $\Gamma$ and $\gamma$, respectively. Accordingly, it can be possibly assumed that all simulated MPCs at each frequency subband rely on UWB multipath channel characteristics [2], [3], [5], [7].

Using this methodology, UWB multipath CIRs and their distorted pulses can be generated based on specific environments. Modeling parameters and types of distortion mechanisms in Table I are selected, corresponding to propagation path cases and related classes of clusters. The height of the transmitter and the receiver is $1.325 \mathrm{~m}$. The time resolution of generated signals in this simulation is 83.32 ps with 6144 data points. Accordingly, the maximum time delay allocating MPCs is approximately $500 \mathrm{~ns}$. However, a very low level of some generated impulse signals is not considered although they appear earlier than $500 \mathrm{~ns}$. The simulation is applied through whole ten frequency-subband received signals with $3 \times 3$ planar arrays. This can lead to complex and congested results ( 81 values for one MPC). Hence, only simulating results observed by the reference $(3,3)$ antenna are presented and used to quantify distortion effects which will be discussed in Section IV.

\section{B. Frequency-Dependent Results}

Referring to dielectric constants of furniture materials in propagation paths [11], properties of dielectric constants with 
TABLE I

TYPICAL VALUES OF MODEl PARAMETERS FOR CIR GENERALIZATION

\begin{tabular}{|c|c|c|c|c|c|c|c|c|c|c|c|}
\hline \multirow{2}{*}{$\begin{array}{l}\text { Scenario } \\
\text { Cases }\end{array}$} & \multirow{2}{*}{$\begin{array}{c}\text { Dominant Cluster } \\
\text { Classes/ Distortion Mechanisms }\end{array}$} & \multicolumn{10}{|c|}{ Simulating Channel Parameters } \\
\hline & & $\begin{array}{l}\text { Cluster } \\
\text { No, } L\end{array}$ & $\begin{array}{l}\text { MPC } \\
\text { No, } K\end{array}$ & $\beta_{\phi}$ & $\beta_{\theta}$ & $\beta_{\tau}$ & $\gamma_{\phi}, \gamma_{\theta}$ & $\gamma_{\tau}$ & $\tau_{0}$ & $\gamma$ & $\Gamma$ \\
\hline $\begin{array}{l}\text { LOS-A } \\
(3-18 \mathrm{~m})\end{array}$ & $\begin{array}{c}\text { Class-III / GO, M-reflection, } \\
\text { Half-plane diffraction }\end{array}$ & $\mathrm{U}[1,5]$ & $\mathrm{U}[1,3]$ & $\mathrm{U}[0,5]$ & $\mathrm{U}[0,5]$ & $\mathrm{U}[0,2]$ & 0 & 0 & $\mathrm{U}[10,60]$ & 4.3 & 7.1 \\
\hline $\begin{array}{l}\text { NLOS-B } \\
(3-18 \mathrm{~m})\end{array}$ & $\begin{array}{l}\text { Class-III / GO, M-reflection, Half-plane } \\
\text { diffraction, Thin slab diffraction }\end{array}$ & $\mathrm{U}[3,7]$ & $\mathrm{U}[1,5]$ & $\mathrm{U}[5,10]$ & $\mathrm{U}[0,5]$ & $\mathrm{U}[0,20]$ & $\mathrm{U}[-0.1,0]$ & 0 & $\mathrm{U}[10,60]$ & 6.7 & 5.5 \\
\hline \multirow{2}{*}{$\begin{array}{l}\text { NLOS-C } \\
(9-30 \mathrm{~m})\end{array}$} & $\begin{array}{l}\text { Class-III / GO, M-reflection, } \\
\text { Half-plane diffraction }\end{array}$ & $\mathrm{U}[3,7]$ & $\mathrm{U}[1,5]$ & \multirow{2}{*}{$\mathrm{U}[10,30]$} & \multirow{2}{*}{$\mathrm{U}[0,5]$} & \multirow{2}{*}{$\mathrm{U}[10,40]$} & \multirow{2}{*}{$\mathrm{U}[-0.1,0]$} & \multirow{2}{*}{0} & \multirow{2}{*}{$\mathrm{U}[30,100]$} & \multirow{2}{*}{7.9} & \multirow{2}{*}{14} \\
\hline & $\begin{array}{c}\text { Class-I+II / Thin slab diffraction, } \\
\text { Thick slab reflection } \\
\end{array}$ & $\mathrm{U}[5,10]$ & $\mathrm{U}[5,10]$ & & & & & & & & \\
\hline $\begin{array}{l}\text { NLOS-D } \\
(>18 \mathrm{~m} .)\end{array}$ & $\begin{array}{c}\text { Class-I+II / } \\
\text { Thick slab reflection }\end{array}$ & $\mathrm{U}[5,10]$ & $\mathrm{U}[5,10]$ & $\mathrm{U}[30,50]$ & $\mathrm{U}[0,5]$ & $\mathrm{U}[20,80]$ & $\mathrm{U}[-0.1,0]$ & 0 & $\mathrm{U}[60,200]$ & 12 & 24 \\
\hline
\end{tabular}

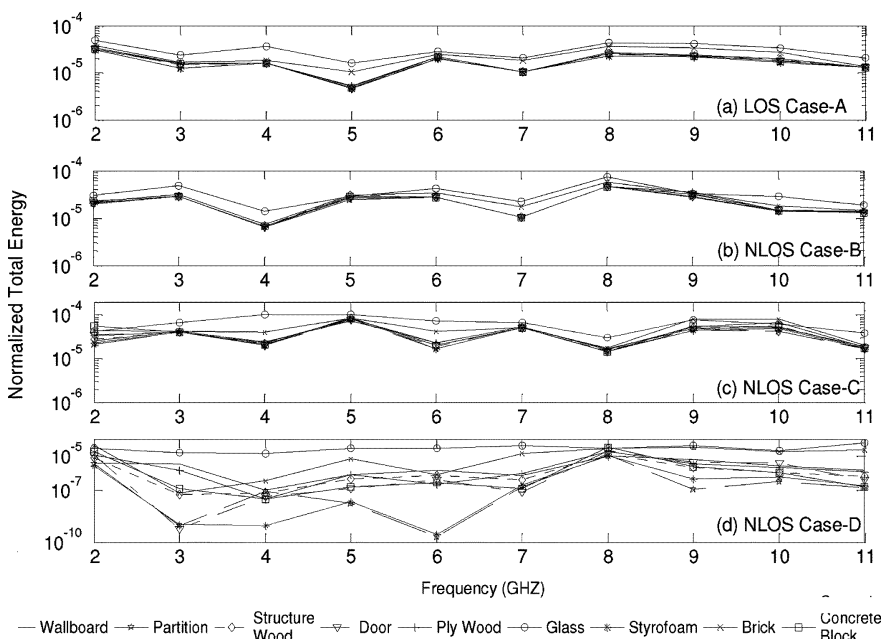

Fig. 2. Comparison of total energy variations of received signals simulated for all frequency subbands.

frequency variation are also taken into account; thus, the simulation at each subband examines pulse distortions from different dielectric constants. The effects of frequency-dependent dielectric constants of materials for individual UWB propagation paths are characterized and their results are shown in Fig. 2. Total energies can be computed by summing up all power of simulated MPCs at each frequency subband. Power in each MPC is normalized over the total power for each obstruction material. It can be seen that there are frequency-selective characteristics for propagation through each material as total energy variations fluctuate nearly the same in the overall subband. But there is dramatic variation of total energies in NLOS Case-D propagation channels. This might be due to various obstructions along the long distance of propagation paths which can distort and attenuate the simulated signal strength.

Furthermore, when considering UWB energy distribution of all UWB channels distorted by various obstructions over all frequency subbands, energy variation classified by distortion mechanisms can be determined as illustrated in Fig. 3. The summation of total energies of all MPCs from all clusters distorted by particular obstructions is calculated. Total energies for MPCs of LOS and GO ray mechanism are less than others. This is caused by the least total numbers of all LOS contributions to be calculated although its received signal energies

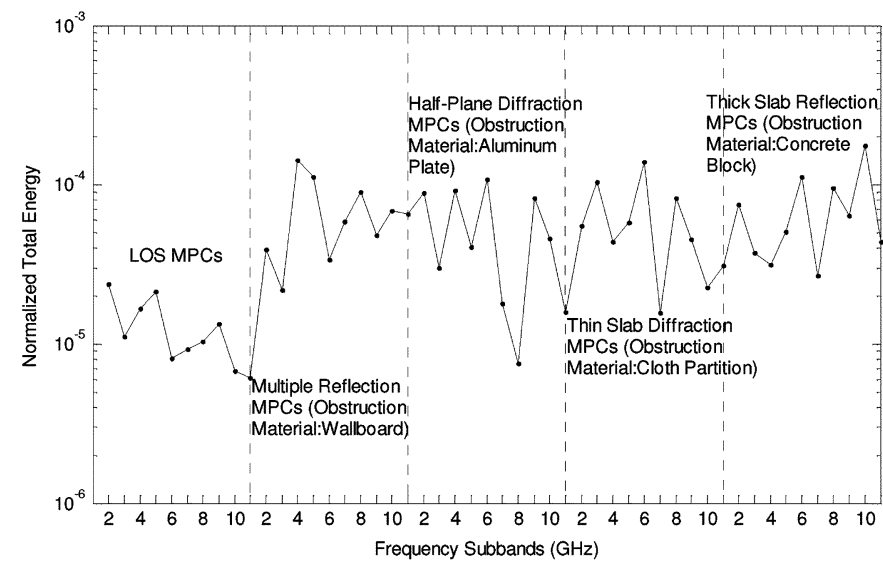

Fig. 3. Frequency-dependent energy distribution of all scenario cases classified by types of distortion mechanism.

are maximum when considering individual path cases. There are the highest normalized total energies in received signals distorted by other mechanisms, such as multiple reflections, half-plane diffractions, thin-slab diffractions, and thick-slab reflections. Due to the large numbers of clusters and MPCs as indicated in Table I, energy distributions of these distortion effects are approximately in the same high level, especially for thin- and thick-slab distortions.

\section{QUANTIFICATION OF Distortion EFFECTS ON SYSTEM PERFORMANCE}

To quantify pulse distortion impacts on UWB system performance, the probability of bit-error rate (BER) is also presented in this section. Pulse position-modulation time-hopping UWB (PPM-TH-UWB) is generated as the transmitted signal conveying $3000 \mathrm{~b}$ through 3000 pulses, thus code repetition coding is not applied. The average pulse repetition period is $60 \mathrm{~ns}$, guaranteeing the absence of intersymbol interference (ISI) in LOS. These generated signals $W(\tau)$ are simulated and transmitted over the synthesized multipath channels as stated in Section II; therefore, CIRs, $h(\tau)$, including cluster types and distortion effects, are taken into account. An ideal RAKE receiver that processes all multipath contributions is simulated for the receiving system. Consequently, the probability of BER $\mathrm{Pr}_{b}$ of received signals $R(\tau)=W(\tau) \otimes h(\tau)$ can be identified by 


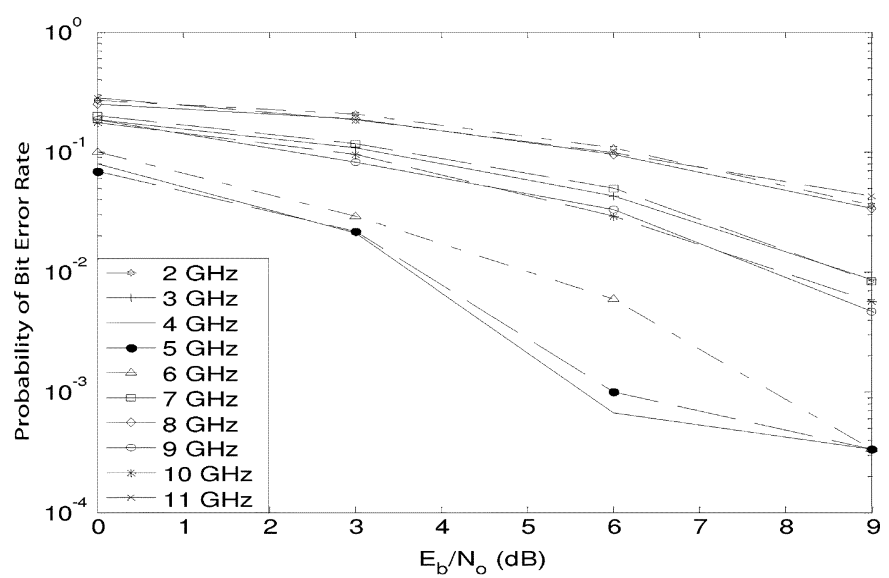

Fig. 4. Comparison of error probability simulated for the LOS Case-A over all frequency subbands.

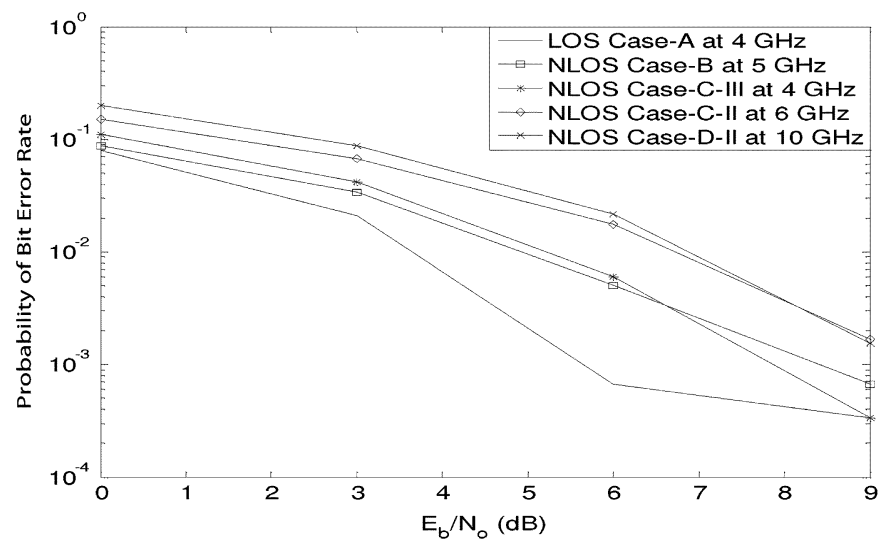

Fig. 5. Comparison between the highest system performances of each scenario.

$$
\operatorname{Pr}_{b}=\frac{1}{2} \operatorname{erfc}\left(\sqrt{\frac{E_{b R X}}{2 N_{0}}}\right)=\frac{1}{2} \operatorname{erfc}(\sqrt{\mathrm{SNR}})
$$

where error function $\operatorname{erfc}(x)=2 / \sqrt{\pi} \int_{x}^{\infty} \exp \left(-x^{2}\right) \mathrm{dx}$ and $E_{b R X}$ represent the received energy per bit [12]. The error probability results in distorted UWB multipath channels for Case-A are illustrated in Fig. 4 where system performances are simulated at all frequency subbands. Comparing performances simulated all subbands, the best performance could be obtained for UWB multipath channels simulated over 4-5 GHz subbands. Furthermore, comparing $\operatorname{Pr}_{b}$ between each scenario case can quantify distortion effects corresponding to cluster classifications and propagation scenarios as depicted in Fig. 5. The best performances of each case allocated in different frequency subbands are compared. Results can be confirmed that distortion effects can degrade system performances regarding characteristics of propagation paths with the highest performance in Case-A followed by Case-B, Case-C, and Case-D, respectively.

\section{CONCLUSION}

This letter presents significant results of the frequency-dependent characteristics and the quantification of distortion effects on UWB multipath channels. The modified simulation, which extends the standard IEEE UWB CIR model with physics-based distortion mechanisms suitably for specific scenarios and cluster types, are proposed and applied to gain the results. Simulation over ten frequency subbands can lead to variation of frequency-dependent normalized energy regarding scenario cases, cluster classification, and obstruction materials. Reasonable investigated results are presented as the maximum total energy distribution and the highest system performance can be observed in the LOS case. In contrast, propagation between rooms gains the worst results of energy distribution and system performance.

\section{REFERENCES}

[1] A. Saleh and R. A. Valenzuela, "A statistical model for indoor multipath propagation," IEEE J. Sel. Areas Commun., vol. 5, no. 2, pp. 128-137, Feb. 1987.

[2] C. Chong, Y. Kim, and S. S. Lee, "A modified S-V clustering channel model for the UWB indoor residential environment," in Proc. IEEE Vehicular Technology Conf., Stockholm, Sweden, May 2005, pp. 58-62.

[3] Q. H. Spencer, B. D. Jeffs, M. A. Jenson, and A. L. Swindlehurst, "Modeling the statistical time and angle of arrival characteristics of an indoor multipath channel," IEEE J. Sel. Areas. Commun., vol. 18, no. 3, pp. 347-359, Mar. 2000.

[4] Y. Chen and V. K. Dubey, "Dynamic simulation model of indoor wideband directional channels," IEEE Trans. Veh. Technol., vol. 55, no. 2, pp. 417-430, Mar. 2006.

[5] K. Haneda, J. Takada, and T. Kobayashi, "Cluster properties investigated from a series of ultrawideband double directional propagation measurements in home environments," IEEE Trans. Antennas Propag., vol. 54, no. 12, pp. 3778-3788, Dec. 2006.

[6] R. J. M. Cramer, R. A. Scholtz, and M. Z. Win, "Evaluation of an ultrawide-band propagation channel," IEEE Antennas Propag., vol. 50, no. 5, pp. 561-570, May 2002.

[7] A. F. Molisch, B. Kannan, C. C. Chong, S. Emami, A. Fort, J. Karedal, J. Kunisch, H. Schantz, U. Schuster, and K. Siwiak, IEEE 802.15.4a Channel Model-Final Rep., San Antonio, TX, IEEE 802.15-04-0662-00-004a, Nov. 2004.

[8] C. Chong, C. Tan, D. I. Laurenson, S. McLaughlin, M. A. Beach, and A. R. Nix, "A new statistical wideband spatio-temporal channel model for 5-GHz band WLAN systems," IEEE J. Sel. Areas Commun., vol. 21, no. 2, pp. 139-150, Feb. 2003.

[9] R. C. Qiu, "A generalized time domain multipath channel and its application in ultra-wideband (UWB) wireless optimal receiver-Part III: System performance analysis," IEEE Trans Wireless Commun., vol. 5, no. 10 , pp. 2685-2695, Oct. 2006.

[10] R. C. Qiu, "A generalized time domain multipath channel and its application in ultra-wideband (UWB) wireless optimal receiver-Part II: Physics-based system analysis," IEEE Trans Wireless Commun., vol. 3, no. 6, pp. 2312-2324, Nov. 2004.

[11] A. Muqaibel, A. Safaai-Jazi, A. Bayram, A. M. Attiya, and S. M. Riad, "Ultrawideband through-the-wall propagation," Proc. Inst. Elect. Eng., Microw. Antennas Propag., vol. 52, no. 6, pp. 581-588, Dec. 2005.

[12] M.-G. D. Benedetto and G. Giancola, Understanding Ultra Wide Band Radio Fundamentals. Upper Saddle River, NJ: Prentice-Hall, 2004, ch. 8 . 